\title{
PHYSIQUE DES SOLIDES ET MORPHOLOGIE DES SURFACES
}

\author{
J. RousSEAU \\ Laboratoire de Tribologie et de Dynamique des Systèmes, URA CNRS 855 \\ Ecole Nationale d'Ingénieurs de Saint Etienne, France \\ et T.G. Mathia \\ Laboratoire de Tribologie et de Dynamique des Systèmes, URA CNRS 855 \\ Ecole Centrale de Lyon, France
}

PHYSICS OF SOLIDS AND MORPHOLOGY OF SURFACES: The morphology of surfaces is now an important field of research because of its direct connection with the industrial activity like manufacturing and optimising of functional criteria through complicated interfacial phenomena. Presently, characierization of the surface morphology, via the profile metrology, is well modelled by a statistical description. The use of shape morphological parameters allows to identify features of the surface structures generated by the process techniques and the emergence of the different phases of the condensed matter. Starting from the solid state background knowledge, prediction of the surface morphology appears as a tedious way. However, progress in the science of the solids formation and industrial requirements promises the best future for the physicists in such a new technological activity. Philosophy and basic formalism of that approach is presented here.

PACS numbers: 68.35.-p, 68.55.Jk

\section{Qu'est ce qu'une surface?}

Tout objet solide est identifié et valorisé par sa surface, soit par son aspect auquel nos sens vue et toucher sont sensibles, soit par sa fonction dans ses usages industriels, soit par les deux. Il doit être vendu: sa surface doit donc attirer et plaire. Il est fonctionnel: sa surface doit donc posséder les propriétés nécessaires à l'usage demandé. L'obtention de ces qualités de surface exige des traitements appropriés et doit aussi être contrôlée.

Communément le terme surface s'attache aux limites d'un objet et aux apparences visuelles (couleur, éclat) et tactiles (formes, relief) de ces limites.

En adoptant un point de vue plus scientifique, on peut identifier deux approches, presque deux écoles séparées par leur finalité et leurs procédures d'investigation. 
- L'une habituellement qualifiée de physique des surfaces s'attache à la détermination de la composition chimique, des interactions et des effets dynamiques mettant en cause les toutes premières courbes atomiques de surface d'un matériau et souvent la seule première couche et les espèces fixées sur cette couche.

- L'autre s'attache à la mesure de la rugosité, à la détermination de la morphologie de la surface des objets manufacturés résultant à la fois de la nature du matériau et de son procédé d'élaboration.

Si ce deuxième aspect constitue l'objet de notre démarche, nous savons que les performances d'usage d'une surface dépendent fortement de son état morphologique mais aussi des acquis de la première approche.

L'établissement des liens entre la physique atomique et l'état morphologique des surfaces après leur élaboration est certes un objectif ambitieux et lointain. Néanmoins, la recherche actuelle s'engage dans cette voie, dégageant déjà quelques pistes, car la ma îtrise des procédés d'élaboration et de caractérisation des surfaces des matériaux, à des échelles de plus en plus petites, conditionne les usages industriels d'avenir. L'ensemble de cette problématique abordée ici constitue un sujet stratégique par sa position scientifique et ses implications économiques.

D'un point de vue formel, les fonctions d'usage des surfaces exigent une description déterministe par des paramètres que l'on peut extraire de trois ensembles de propriétés (physico-chimiques, rhéologiques et morphologiques) de la matière condensée. Selon le rôle demandé à une interface, se pose la question de la sélection d'un nombre limité de paramètres pertinents et accessibles par les techniques d'analyse. La solution se situe dans le domaine commun des trois ensembles de propriétés.

Dans ce qui suit, nous nous limitons à une contribution naïve décrivant quelques liens qui existent entre la physique du solide et la morphologie des surfaces.

\section{Positionnement du sujet}

\subsection{Sujet stratégique}

\subsubsection{Un argument scientifique}

L'état morphologique des surfaces devient un thème scientifique de plus en plus travaillé, si l'on en juge par les publications et les demandes de coopération internationale qui s'y rapportent. Après un déclin relatif de cinq à huit ans, on observe une reprise d'intérêt vraisemblablement liée au développement des instruments d'analyse, en particulier subnanométriques, et à la découverte de matériaux à propriétés spéciales: super réseaux, supraconducteurs, interfaces de jonction, matériaux à mémoire.

L'importance des recherches accordées aux surface suit celle de la physique du solide, c'est pourquoi les physiciens doivent maintenant s'investir davantage dans les études de morphologie des surfaces et des propriétés fonctionnelles liées à cette caractéristique du solide. En effet, dans l'approche scientifique dominante, la morphologie des surfaces est une caractéristique de la matière dense relevant très largement d'une description mécanistique, ce qui apparaît logique tant que 
cette notion reste considérée comme une propriété continue de la matière. Mais les progrès scientifiques et techniques permettent maintenant d'élaborer des surfaces solides, y compris pour des produits industriels, dont la morphologie doit être contrôlée à des échelles subnanométriques. A ces échelles de quelques $\AA$, le concept de continuité, au sens usuel de la mécanique des solides, ne peut être conservé. Le comportement de la matière et son organisation géométrique et morphologique relèvent progressivement de son caractère discret plus familier aux physiciens du solide.

\subsubsection{Un argument économique}

La morphologie des surfaces joue un rôle important dans la plupart des phénomènes classiques de tribologie tels que lubrification et frottement à sec, mais aussi influence de nombreux phénomènes physiques comme les conductivités électrique et thermique, l'adhésion, le mouillage, ... Les moyens financiers engagés dans la progression de la connaissance et la maîtrise de ces phénomènes sont colossaux. Considérons, à titre d'exemple, le cas d'un moteur. Les parties et les mécanismes faisant l'objet des préoccupations des tribologues sont nombreux. Nous n'en examinerons qu'un: l'association vilebrequin-paliers de roulement qui représente une dépense mondiale annuelle de plusieurs millions de francs uniquement en lubrification et usure. Que faire pour que ce système ne s'use pas? Comment réduire l'abrasion par frottement? Voici deux questions qui sont dans la thématique générale du sujet considéré ici.

La notion de contrôle qualité qui pénètre de plus en plus dans le secteur de la production industrielle est un puissant stimulant des études de morphologie des surfaces qui font le lien entre la production et la recherche. D'une façon très générale, il appara ît maintenant nécessaire de réfléchir sur le contexte socio-économique sous jasent à une technique ou à une recherche avant de décider d'investir dans cette technique ou cette recherche.

\subsection{Emergence du concept de forme et morphologie moderne}

\subsubsection{Rappel sur la perception visuelle des formes.}

Toute observation morphologique aboutit en définitive à une observation visuelle humaine dont le problème central est la reconstruction perspective des formes et le repositionnement des objets dans l'espace continu tridimensionnel à partir des images rétiniennes bidimensionnelles et digitales. Cette reconstruction s'opère selon le mécanisme de la perception visuelle des formes connu depuis 1982 [1]. L'idée de base, à retenir ici, est que la reconnaissance d'une forme et son positionnement partent des projections de cette forme, selon des directions différentes, sur les cellules sensibles de la rétine (Fig. 1). A partir des différents "contours apparents" bidimensionnels ainsi crés, le cerveau reconstitue la forme par comparaison au "vécu" de l'observateur.

Le processus de vision d'un objet par l'oeil se ramène donc à un traitement de l'information véhiculée par un signal lumineux allant de l'objet à l'oeil. En préalable à ce traitement se pose donc la qualité du contenu physique du signal. Définir intrinsèquement et objectivement la morphologie d'un objet implique que 


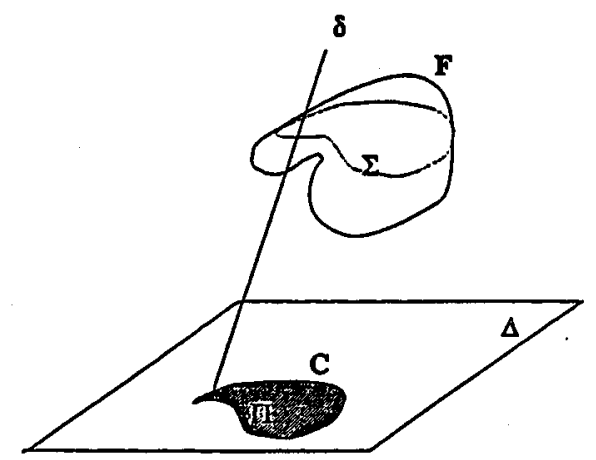

Fig. 1. Principe de la projection rétinienne dans le mécanisme de la vision: $F$ - forme matérielle présentant une surface régulière $S$ dạns $R^{3}, \Delta$ - plan de projection assimilé à un plan de cellules rétiniennes, $\delta$ - direction de projection. Pour l'application $P$ de $R^{3}$ sur $\Delta$ parallèlement à $\delta$, on peut définir le contour $C=P(S)$ dans $R^{2}$, et le contour $\Sigma=$ points de $S$ où $\delta$ est tangente à $S$ \} qui est le générateur de $C$.

l'on comprenne comment s'inscrit dans le signal lumineux la géométrie des contours apparents révélatrice d'une discontinuité physique. C'est un aspect complexe de l'interaction onde électromagnétique-matière, puisque les singularités détectées par la vision de l'observateur en termes d'optique géométrique ont été générées en tant que phénomènes d'optique ondulatoire; en particulier lorsque l'observation correspond à de très petites échelles de distance.

\subsubsection{Incompalibilité fondamenlale avec l'objectivilé physique mécanistique}

Le monde auquel nos sens sont sensibles est constitué de formes naturelles. Toutefois, jusqu'à une époque récente, la science de la morphologie n'existait pas, puisque c'est seulement à partir de 1960 que l'on a commencé à comprendre et élaborer les concepts permettant de structurer objectivement les formes naturelles. Le but consistait à développer une théorie des formes, compatible et si possible réductible à des contenus pliysiques. La difficulté résulte du concept moderne d'objectivité physique qui, de nature mécanistique (Newton), a engendré le développement d'une modélisation physico-mathématique des forces. Le succès de cette modélisation a créé un fantastique obstacle à une théorie des formes. En effet, la conception physique de la matière, développée comme une mécanique d'interaction entre particules, tend à inculquer aux esprits l'évidence de l'irréductibilité des formes à une physique de la matière.

Pour combattre et inverser cette tendance profonde de la pensée scientifique, les formes doivent être décrites de façon intrinsèque en tant que constituants de notre perception du monde extérieur, bien qu'elles offrent une très grande variété physique et biologique et soient attachées à des phénomènes qui aident à leur compréhension. L'avancée scientifique en ce sens est réalisée dans la formalisation moderne récente de la morphologie s'appuyant sur une conceptualisation de la forme intégrée aux problématiques topologiques et physiques de la plus grande actualité. 


\subsubsection{Approche topologique intrinsèque}

La structure cristalline réelle des solides se présente comme une juxtaposition de grains de taille variables. Chaque grain se distingue de ses voisins par au moins une qualité: orientation, propriétés mécaniques ou physiques, composition chimique, ... Toute approche topologique pure doit pouvoir rendre compte d'une telle qualité distinctive. La démarche suivie pour parvenir à cet objectif part du concept qu'une forme sensible $F$, identifiée dans l'espace extérieur $E$ (espace substrat), occupe une certaine portion $D$ de $E$ limitée par un bord $B$. Ce domaine d'occupation contient des qualités sensibles $q_{1} \ldots q_{n}$, ou grandeurs intensives $q_{i}(D)$, par opposition à la qualité première qui est l'extension spatiale. La forme doit être phénoménologiquement perçue par contraste de propriétés d'où l'importance de la notion de discontinuité qualitative qu'on doit lui affecter. En se référant au traitement de Thom [2], on est amené à distinguer deux types de points dans $D$ :

- les points réguliers possédant un voisinage où les $q_{i}$ varient continûment et engendrent un ouvert $U$ de $D$ tel que si $D$ appartient à $U$ le substrat est homogène;

- les points singuliers qui forment un fermé $K^{*}$ de $D$ complémentaire de $U$. Si $P$ appartient à $K$, le substrat est localement hétérogène en $P$.

Comprendre et analyser l'origine de cette hétérogénéité fait l'objet de la théorie des catastrophes en physique. Parallèlement à cette théorie se développe une approche purement mathématique décrivant, d'une part la structure géométrique effective d'une forme, d'autre part son voisinage [3].

\subsubsection{Approche physique de la création des formes}

De façon très générale, un système naturel est organisé à deux niveaux de réalité:

- un niveau microscopique caché el discontinu, décrit par la modélisation mécanistique des interactions entre ses composants discrets,

- un niveau macroscopique visible et continu pour nos sens auquel s'applique la description morphologique.

L'organisation de la matière, continue d'apparence, est la manifestation, l'émergence, des processus d'interactions élémentaires du niveau microscopique. Les morphologies observées dépendent donc de la microphysique, par l'intermédiaire de phénomènes tels que: transitions de phase, germinations, croissances, ségrégations, transitions vitreuses ...

A titre d'exemple on peut considérer un phénomène de transition de phase. C'est un cas évident d'auto-organisation de la matière élémentaire au passage d'une valeur critique d'un paramètre de contrôle (la température généralement). Le système physique change brusquement d'état thermodynamique et de qualités macroscopiques. Il présente une discontinuité qualitative, émergence de la physique "fine", bien illustrée par le cas standard de la transition magnétique "ferro-para". Il est bien connu qu'au de là d'une température critique $T_{C}$ (point de Curie), un composé ferromagnétique perd son aimentation spontanée et devient paramagnétique. Landau a donné une première interprétation de cet effet en introduisant un paramètre d'ordre $n(r), r$ définissant la position d'un point du substrat. Ce paramètre est une variable extensive, conjuguée de la variable intensive champ 
magnétique $H$, devenant nulle au passage de l'état ferromagnétique ordonné à l'état paramagnétique désordonné. Au point critique, le comportement du système est déterminé par les "exposants critiques". Ceux-ci permettent d'exprimer les grandeurs thermodynamiques caractéristiques du système sous forme de fonctions analytiques faisant intervenir les puissances de $\left(T-T_{\mathrm{C}}\right)$ ou de $n(r)$. Dans l'approximation du premier ordre de Landau, la densité d'énergie libre $F(n)$ du système présente des oscillations, ou parties singulières, lorsqu'on fait tendre le volume du système vers l'infini. Ces fluctuations deviennent macroscopique au voisinage du point critique et génèrent des pics dans la fonction de partition du système identifiables à des catastrophes élémentaires. En outre, une propriété caractéristique du système: la longueur de corrélation diverge au point critique. Le système devient invariant par changement d'échelle et l'on peut donc considérer le point critique comme un point fixe pour les transformations d'échelle. Ces remarques sont à l'origine de l'introduction du groupe de renormalisation par Kadanoff.

Les phénomènes critiques manifestent de grandes propriétés d'universalité avec, d'une part des comportements critiques typiques observables au niveau macroscopique, mais d'autre part une certaine autonomie vis à vis de la physique fine des substrats; par exemple, lorsque le phénomène critique dépend de la dimension du système comme dans le cas précédent.

A partir de cet exemple illustrant un comportement très général de la matière condensée, on voit qu'un système naturel, au niveau macro, s'organise autour des singularités de la physique micro qui supportent l'information et sont phénoménologiquement dominantes. C'est l'infrastructure catastrophique qu'elles engendrent qui émerge au niveau supérieur de la perception visuelle directe ou assistée par les instruments d'observation.

Identifier et comprendre, comment les phénomènes élémentaires critiques régissant l'organisation intime de la matière condensée se manifestent dans la morphologie des surfaces, est un objectif scientifique difficile à cause de la diversité et de la complexité de ces phénomènes. Il sera abordé dans Sec. 3 après avoir introduit les concepts de base de la morphologie des surfaces.

\section{Etat morphologique de surface des matériaux solides}

\subsection{Notions de base de la morphologie des surfaces}

La morphologie d'une surface se manifeste à travers les différents éléments constitutifs que l'on a été amené à considérer [4]:

- le motif qui apparaît comme l'élément fondamental, isolé ou répété, donnant l'aspect spécifique de la surface. Il peut comprendre une ou plusieurs traces ou empreintes, en creux et en saillies;

- la structure: qui est constituée par l'arrangement des motifs;

- la texture: qui se rapporte à la disposition et l'arrangement des phases (ou des parties) constitutives de la surface, en particulier dans le cas de matériaux non homogènes.

L'identification de ces constituants exige des moyens de contrôle et d'observation dépendant de l'échelle d'analyse recherchée et que l'on peut prendre en compte 
à partir de la notion de profil topographique vis à vis de la fonction finale d'usage recherchée pour une surface. Compte tenu de la diversité morphologique d'une surface, sa caractérisation quantitative pose des problèmes mathématiques et métrologiques indépendamment de l'interprétation physique du résultat. Si le contenu d'un profil constitue un ensemble d'informations topologiques suffisantes, son analyse permet de caractériser convenablement une surface. Cette approche ne peut être retenue que pour des surfaces isotropes.

\subsection{Notion de profil topographique stationnaire}

Nous ne considérerons ici que la caractérisation de la topographie stationnaire de surface. De plus, nous admettrons que le dispositif de scrutation a une taille infiniment petite par rapport aux aspérités du relief, ce qui correspond à une observation du profil sans déformation. Partant des mesures métrologiques, toute connaissance de l'état morphologique d'une surface repose sur une analyse topographique des étalements dans les sens vertical et horizontal et sur une caractérisation tridimensionnelle:

\subsubsection{Méthode générale de description}

L'état topographique d'une surface peut être défini par la fonction $f(x, y, z)$ en tous points de celte surface. Une telle définition implique la localisation et l'utilisation d'un système de référence lié ou non à la surface à caractériser. Pour $N$ surfaces analogues de même forme constituées d'un même matériau et obtenues dans des conditions de génération identiques, la hauteur $z(x, y)$ peut être considérée comme une variable aléatoire. L'ensemble des hauteurs $z(x, y)$ constitue une certaine distribution statistique, dont les différents moments et fonctions caractéristiques sont des moyennes d'ensemble.

En pratique, au lieu d'effectuer ces moyennes d'ensemble sur les surfaces de $N$ échantillons, on n'explore qu'une portion de surface d'une seule éprouvette. Cette procédure offre toute satisfaction lorsque ces moyennes ne dépendent pas de la portion explorée, ce qui est le cas des surfaces dites homogènes-aléatoires. En général, une surface ne présente pas cet état idéal et se compose de motifs (éléments fondamentaux analogues) arrangés selon une ou plusieurs tendances. L'arrangement de ces motifs forme la structure de la surface. Il résulte à la fois des procédés d'élaboration et d'usure des pièces qui possèdent leur signature, mais également de la constitution interne propre du matériau. On classe les surfaces selon leur mode d'élaboration et leur structure: homogène, hétérogène ou anisotrope etc.

Dans tous les cas, avant de caractériser une surface à partir de la mesure de ses profils, se pose la question de l'évaluation de l'ergodicité (pour les surfaces supposées homogènes-aléatoires) et de la stationnarité des résultats. La vérification de ces propriétés est extrêmement délicate car, a priori, les résultats obtenus sont fonctions des dimensions des portions de surface étudiées. Il est donc indispensable de procéder, dans un premier temps, à une exploration tridimensionnelle de la surface entière, puis de caractériser l'étalement vertical et horizontal d'un profil selon plusieurs directions et dans différentes zones de la surface. On pourra alors vraiment déterminer l'isotropie et l'homogénéité de la surface. 


\subsubsection{Analyse de l'élalement vertical d'un profil de surface}

L'étalement vertical d'un profil peut être représenté par la hauteur de ses différents points par rapport à une ligne de référence. En considérant le profil $z(x)$, dans la direction $x$, comme une variable aléatoire sur une longueur mesurée $L$ suffisamment grande, la probabilité de trouver un point du profil, entre les hauteurs $z$ et $z+\mathrm{d} z$, est donnée par

$$
\operatorname{Prob}[z<z(x)<z+\mathrm{d} z]=\lim _{L \rightarrow \infty} l_{z} / L,
$$

où $l_{z}$ est la longueur du profil parcouru le long de la ligne droite de référence dont l'ordonnée est comprise entre $z$ et $z+\mathrm{d} z$. Quand $\mathrm{d} z$ est suffisamment petit et $L$ suffisamment grand, on peut définir la fonction densité de probabilité $p(z)$ des ordonnées

$$
p(z)=\lim _{\mathrm{d} z \rightarrow 0}\{\operatorname{Prob}[z<z(x)<z+\mathrm{d} z]\} / \mathrm{d} z=\lim _{\mathrm{d} z \rightarrow 0}(1 / \mathrm{d} z) \lim _{L \rightarrow \infty}\left(l_{z} / L\right) .
$$

A l'aide de cette statistique descriptive, on s'efforce de donner un résumé quantitatif d'une distribution des ordonnées du profil mesuré. Suivant le type d'informations recherchées, on distingue trois classes de paramètres:

- les paramètres de position, qui permettent de situer une distribution, par une valeur sur une échelle de mesure. Les plus connus sont: la moyenne, la médiane, le mode, les moments non centrés, etc.

- les paramètres de dispersion, qui caractérisent l'homogénéité de la distribution et comprennent: l'étendue, la variance, l'écart-type, les moments centrés, etc.

- les paramètres de forme, qui permettent d'apprécier l'asymétrie et la fincsse d'une distribution.

Si l'on dispose d'un nombre suffisant de données se rapportant à une même population, on peut étudier la distribution de la variable aléatoire $z(x)$ à l'aide des moments centrés. Le moment centré d'ordre $q\left(\mu_{q}\right)$ est donné par l'expression suivante:

$$
\mu_{q}=\int_{-\infty}^{+\infty}(z-z)^{q} P(z) \mathrm{d} z
$$

dans laquelle $z$ est la valeur moyenne de $z$. A l'aide des moments centrés d'ordre 2,3 et 4 , on peut exprimer les deux principaux paramètres de forme $\beta$ et $\gamma$ qui caractérisent assez bien, la typologie de nombreuses surfaces industrielles résumée dans Tableau [5]:

- le paramètre $\beta_{2}$ ou coefficient d'aplatissement de Pearson (ou Kurtosis): traduit la finesse de la distribution. Une valeur élevée exprime une faible dispersion des valeurs et une forte stabilité du profil autour du niveau de plus grande probabilité (cf. profils schématiques insérés dans Fig. 3);

- le paramètre $\gamma_{1}$ ou coefficient d'obliquité de Fisher (ou Skewness): caractérise l'asymétrie de la distribution des hauteurs de profils. La valeur négative de ce coefficient exprime la dominance des nombreux creux, pores et rayures, tandis que le signe positif indique une prépondérance des bosses et des sommets (cf. profils schématiques insérés dans Fig. 3). 
TABLEAU

Profils types et paramètres de forme caractérisant les lois de distribution.

\begin{tabular}{|c|c|c|c|c|}
\hline \multicolumn{4}{|c|}{ Paramètres statistiques } & \multirow[b]{2}{*}{$\begin{array}{c}\text { Critères } \\
\text { industriels }\end{array}$} \\
\hline \multirow{3}{*}{$\begin{array}{l}\text { de } \\
\text { posi- } \\
\text { tion }\end{array}$} & Appellation & Formule & $\begin{array}{c}\text { Distribution } \\
\text { normale }\end{array}$ & \\
\hline & moment d'ordre $q$ & $m_{q}=\int_{-\infty}^{+\infty} Z^{q} p(Z) \mathrm{d} Z$ & - & - \\
\hline & $\begin{array}{l}\text { moment d'ordre } 1 \\
\text { moyenne }\end{array}$ & $m_{1}=\int_{-\infty}^{+\infty} Z p(Z) \mathrm{d} Z=\bar{Z}$ & $\overline{\bar{Z}}$ & $\overline{R_{p}}$ \\
\hline \multirow{5}{*}{$\begin{array}{l}\text { de } \\
\text { dis- } \\
\text { per- } \\
\text { sion }\end{array}$} & moment centré d'ordre $q$ & $\mu_{q}=\int_{-\infty}^{+\infty}(Z-\bar{Z})^{q} p(Z) \mathrm{d} Z$ & - & - \\
\hline & moment centré d'ordre 1 & $\mu_{1}=\int_{-\infty}^{+\infty}(Z-\bar{Z}) p(Z) \mathrm{d} Z$ & $\mathbf{0}$ & - \\
\hline & $\begin{array}{l}\text { moment centré d'ordre } 2 \\
\text { variance }\end{array}$ & $\mu_{2}=\int_{-\infty}^{+\infty}(Z-\bar{Z})^{2} p(Z) \mathrm{d} Z$ & $\overline{\sigma^{2}}$ & - \\
\hline & écart-type & $\sigma=\sqrt{\mu_{2}}$ & - & RMS \\
\hline & écart total étendue & $H_{t}=Z_{i \operatorname{maxi}}-Z_{i \operatorname{mini}}$ & - & $R_{t}$ \\
\hline \multirow{2}{*}{$\begin{array}{l}\text { de } \\
\text { for- } \\
\text { me }\end{array}$} & $\begin{array}{l}\text { coefficient de symétrie } \\
\text { de Fisher }\end{array}$ & $\frac{\mu^{3}}{\sigma^{3}}=\frac{\mu_{3}}{\left(\mu_{2}\right)^{3 / 2}}=\gamma_{1}$ & o & $\gamma_{1}$ \\
\hline & $\begin{array}{l}\text { coefficient } \\
\text { d'aplatissement de } \\
\text { Pearson }\end{array}$ & $\frac{\mu_{4}}{\sigma^{4}}=\frac{\mu_{4}}{\left(\mu_{2}\right)^{2}}=\beta_{2}$ & 3 & $\beta_{2}$ \\
\hline
\end{tabular}

\subsubsection{Analyse de l'élalement horizontal d'un profil de surface}

La fonction d'auto-corrélation $R_{z}(l)$ est la fonction la plus utilisée, dans l'étude de la périodicité du profil. Elle exprime la dépendance ou la liaison pouvant exister entre deux points quelconques du profil, en fonction de la distance $l$ les séparant. Si le profil est purement aléatoire, cette relation est inexistante, donc nulle, sauf pour $l=0$. Si le profil est purement périodique, cette relation est totale, donc égale à $l$, pour toutes les valeurs de $l$ multiples entiers de la période.

L'exploitation de la fonction auto-corrélation reste souvent complexe car on ne peut que difficilement en extraire toute l'information qu'elle contient, sauf lorsqu'on a affaire à des profils facilement identifiables ou lorsque l'on peut émettre des hypothèses valables relatives au mode d'obtention d'une surface et à la structure interne du matériau.

\subsection{Caractérisalion topographique tridimensionnelle (3D)}

L'analyse tridimensionnelle de la surface présente d'importants avantages [6]:

- exploration bidimensionnelle donc entière de la surface,

- archivage graphique et/ou numérique de la surface entière,

- exploitation analytique et traitements appropriés de la section étudiée dans la direction souhaitée,

- localisation de la zone représentative ou particulière de la surface,

— caractérisation du degré d'anisotropie,

- caractérisation du degré d'homogénéité,

- définition de la forme et de la taille des motifs, 
- définition des directions privilégiées.

La caractérisation topographique (3D) s'effectue, dans la plupart des cas, par l'établissement d'une succession de profils selon un algorithme spécifique de l'application envisagée.

\subsubsection{Homogénéité et isotropie des surfaces}

La surface sera considérée comme homogène, si la description statistique demeure invariable par déplacement sur la surface. En ce cas; les paramètres et les fonctions statistiques de la distribution des hauteurs des profils ne dépendent que du vecteur $r$ joignant deux points $P$ et $P^{\prime}$.

Dans le cas où la surface est isotrope, la fonction d'auto-corrélation, ne dépend que du module de $r$ et non de son orientation. Les propriétés statistiques sont indépendantes de la direction du profil.

Par suite de la diversité structurale de la matière, cette classe de surfaces se rencontre très rarement.

\subsubsection{Anisolropie des surfaces}

Dans le cas des surfaces anisotropes, une approche différente doit être mise en place. La connaissance des degrés d'anisotropie d'une surface, l'identificalion des directions privilégiées et de leurs orientations contribuent de manière prépondérante à l'optimisation fonctionnelle des systèmes dans leur entier.

Les aptitudes au collage, à la glisse, à l'écoulement ou à la lubrification, à l'emmanchement etc. sont souvent déterminées par une certaine anisotropie des surfaces rugueuses. Il est donc indispensable, pour de nombreuses fonctions mécaniques, de disposer d'un outil mathématique simple dans son emploi qui offre, aux professions concernées par ces problèmes, la possibilité de déterminer les critères significatifs et de les évaluer facilement. En pratique, on distingue les approches d'anisotropies typologiques, d'étalements vertical et horizontal du profil et morphologiques par le degré d'anisotropie.

La structure interne de la matière, par ses constituants et son mode d'élaboration d'une part, le mode d'élaboration des surfaces d'autre part, déterminent le degré d'anisotropie de la morphogenèse.

\section{Tentative de quantification des morphologies de surface}

\subsection{Génération}

\subsubsection{Séquences}

D'une manière très générale on peut considérer que l'état morphologique de la surface d'un objet manufacturé résulte d'une formation en trois étapes successives:

- En premier, se situe l'élaboration des matériaux qui conduit à différents états structuraux selon le procédé utilisé. Le produit obtenu tient au rôle des "acteurs géophysiques primaires", que sont les liaisons interatomiques et le mode d'empilement des atomes, mais aussi aux "forces motrices" qui orientent la structuration spatiale de la matière condensée: composition chimique, température, phénomènes de transport de matière et de chaleur, etc. 
- Vient ensuite la génération de la surface, selon des techniques de traitement très variables, où interviennent presque toujours une ou plusieurs phases d'usinage et de finition s'effectuant avec des machines outils. Les propriétés mécaniques du matériau, acquises dans l'étape précédente, sont alors sollicitées. Des contraintes mécaniques apparaissent produisant, en retour, des modifications des propriétés physico-chimiques.

- Enfin, l'usure, l'évolution d'usage et le vieillissement des pièces manufacturées qui engendrent des effets combinant, à nouveau, propriétés mécaniques et physico-chimiques dans les phénomènes de morphologie.

Chaque mode de génération des surfaces possède ses caractéristiques (sa signature) qui se manifestent par des éléments identiques dans la morphologie des surfaces. La même déduction s'applique en fonction de la nature des matériaux et des facteurs physico-chimiques permettant de définir cette nature, mais elle peut être rendue difficile par la "signature" des modes de génération ou d'usure. Malgré son importance économique et industrielle cette partie de l'approche quantitative demeure encore très empirique.

\subsubsection{Facteurs génitifs}

On se limite volontairement ici à une approche académique ultrasimplifiée des aspects physiques du processus génitif.

- Forces de liaisons interatomiques et cohésion de la matière

Les différentes forces de liaisons interatomiques se classent en liaisons fortes (ioniques, covalentes, iono-covalentes, métalliques) et en liaisons faibles (liaisons hydrogène et de Van der Waals).

La force de la liaison et son caractère directionnel déterminent les propriétés mécaniques telles que la dureté, la ductilité, la fragilité et la plasticité qui conditionnent ensuite, dans une grande mesure, le comportement des surfaces à l'usinage et à l'usure. Les liaisons ioniques et métalliques sont non directionnelles contrairement aux liaisons covalentes qui sont directionnelles.

A propos du rôle des liaisons faibles dans le comportement des matériaux et la morphologie de surface, on doit retenir: der Waals,

- la transition vitreuse des élastomères qui fait intervenir les liaisons de Van

- la manifestation des liaisons hydrogène dans les propriétés de l'eau et la solidification des chaînes polymériques.

- Empilement des atomes et états condensés de la matière

La formation des liaisons interatomiques provoque la condensation de la matière à l'état liquide comme à l'état solide. C'est pourquoi, la nature des liaisons détermine en partie les propriétés des phases condensées, même si le lien entre la description géométrique microscopique et le comportement macroscopique mécanique n'est pas toujours évident à établir. Ce lien passe par la connaissance des processus d'empilement des atomes, des défauts des structures cristallines et 
de l'évolution de l'état condensé sous l'action des contraintes. La morphologie des surfaces témoigne du jeu complexe de ces différentes catégories de paramètres.

Parmi les phénomènes à prendre en compte pour comprendre la formation des structures morphologiques de surface, on doit retenir:

- les phénomènes de germination et de croissance,

- les transformations de phases,

- les défauts cristallins,

- l'état microstructural obtenu.

Pour illustrer ce dernier phénomène, on peut brièvement examiner l'organisation structurale de différents types de microstructures reproduite schématiquement sur Fig. 2. La compréhension et la prédiction de la morphologie de surface de

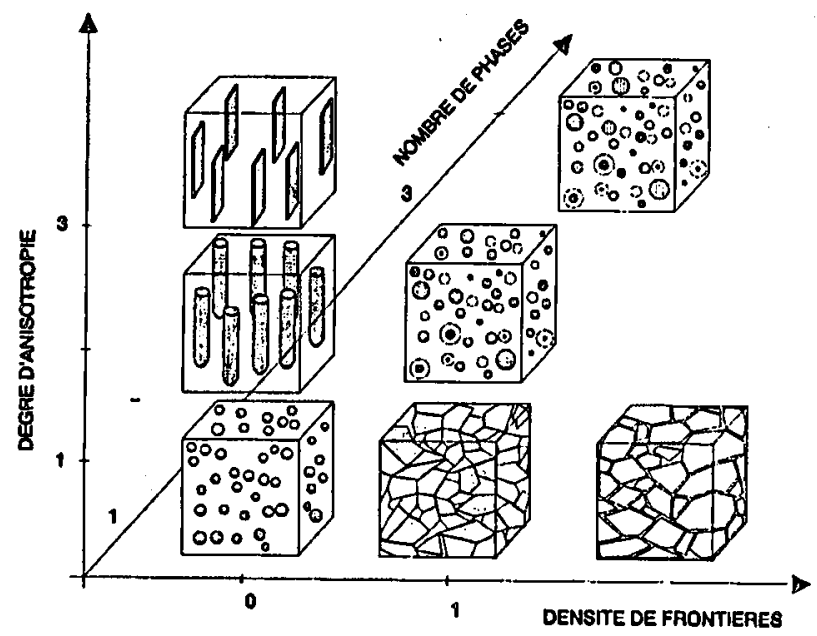

Fig. 2. Représentation symbolique schématique du processus mental de prédiction de la morphologie de surface en fonction de l'état microstructural.

matériaux hétérogènes par leur microstructure demandent une caractérisation du type microstructural [7] (ou classification des hétérogénéités [8]) à partir:

- du degré d'anisotropie prenant en compte la forme, l'orientation et la distribution des microcristallites dans la matrice (degré 1 pour une sphère, degré 2 pour un cylindre, degré 3 pour une plaquette);

- du nombre de phases de leur nature et de leurs fractions volumiques;

- de la densité de frontières définie par la somme des aires interfaciales des grains sur le volume de l'échantillon. Cette grandeur peut être introduite pour tous les grains différant par leur nature chimique ou cristalline.

On dira que la dispersion d'un composé est idéale dans un substrat, lorsque les particules du composé sont isolées les unes des autres el réparties de façon statistiquement régulière dans le volume du substrat.

Malgré un formalisme relativement évolué, tant sur le plan de la physique de la matière que de la topologie, la prédiction de la morphogenèse de surface reste un exercice ambitieux mais envisageable dans le contexte des connaissances. 


\section{2. "Dégénération"}

Avec ce terme nous voulons introduire le concept de l'évolution des surfaces postérieure à leur génération et précédant leur utilisation.

A titre d'exemple, pour illustrer ce qui précède, nous allons considérer, dans l'espace morphologique $(\gamma, \beta)$, les domaines caractéristiques des morphologies de surfaces, mis en évidence par l'un d'entre nous (T. Mathia) avec ses collaborateurs [9], a vec divers alliages et selon différents procédés d'usinage.

\subsubsection{Cas de composés de structure faiblement hétérogène}

Figure 3 présente les résultats obtenus avec les trois surfaces suivantes:

- surfaces de Duralumin (alliage d'aluminium macroscopiquement homogène) générées respectivement par sablage (AS) et par rodage doux (AR),

- surface de fonte grise (matériau macroscopiquement peu hétérogène) produite par rodage abrasif.

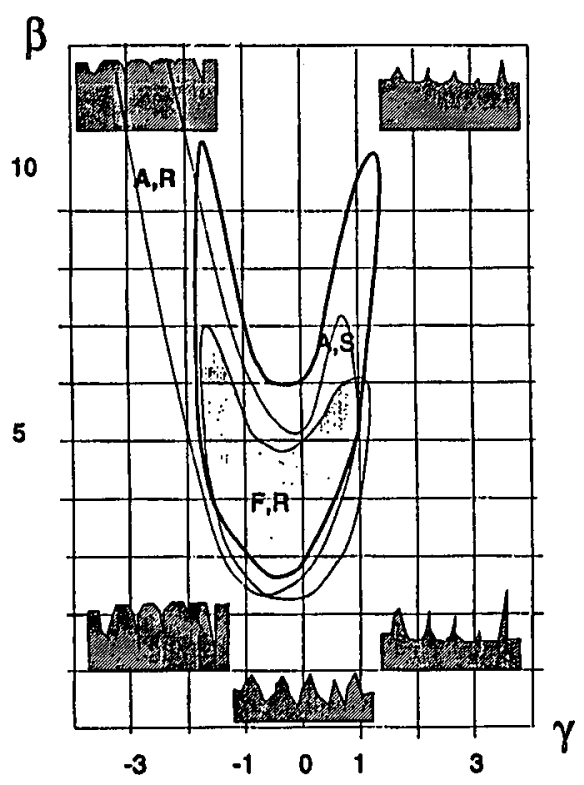

Fig. 3. Représentation des domaines caractéristiques des surfaces de Duralumin générées par sablage (AS) ou par rodage (AR) et de fontes blanches générées par rodage (FR). Les schémas de profils insérés dans les différentes parties de la figure illustrent qualitativement l'allure topographique de la surface en fonction des valeurs des paramètres de forme: Kurtosis et Skewness.

On constate que:

i) le domaine caractéristique du Duralumin dépend du type d'usure imposé lors des différents essais. Le profil de sablage passe aléatoirement du type plateau-pic (gamma $1>1$ ) à plateau-creux $\left(\right.$ gamma $\left._{1}<1\right)$ dans le domaine tandis que le rodage induit une évolution du profil vers une très forte dominance 
du type plateau-creux par suite de l'arasement progressif des parties en relief.

ii) le chemin d'évolution morphologique des fontes grises, au cours du temps induit progressivement un profil dissymétrique du type plateau-creux, comme dans le cas du Duralumin.

\subsubsection{Cas d'un composé de struclure fortement hétérogène}

Figure 4 montre la dispersion des paramètres morphologiques de fontes blanches au chrome-molybdène générées par abrasion (procédé comparable au sablage) dans des conditions rigoureusement identiques. La dispersion des points devient ici totalement aléatoire. En suivant l'évolution d'une surface au cours du temps, on saute de la région plateau-creux à la région plateau-pic sans constater la moindre tendance à la stabilisation sur un type de profil. Ce comporte-

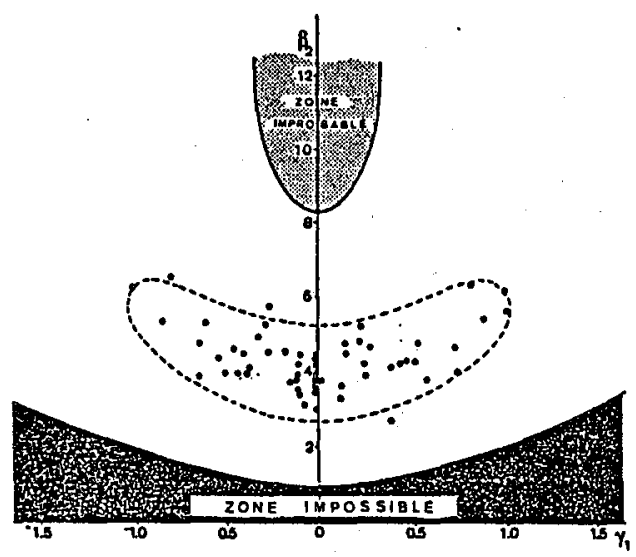

Fig. 4. Représentation du domaine caractéristique des surface de fontes blanches au chrome molybdène, dans l'espace morphologique des paramètres de forme.

ment s'explique aisément en se rapportant au mécanisme de l'usure en surface d'un matériau présentant une forte hétérogéneiité de dureté. Les parties molles s'éliminent pour laisser progressivement pointer les grains plus durs, d'où une tendance vers la création de profils en plateau-pics. Lorsque les grains durs sont suffisamment déchaussés par l'élimination des parties molles, ils sont arrachés par les abrasion, avec production de creux. On génère donc des profils du type plateau-creux, à partir desquels se produit à nouveau l'évacuation des parties molles rendues accessibles à l'érosion. L'entretien de ce processus rend bien compte de la dispersion aléatoire des points, dans l'espace morphologique.

En considérant simultanément les résultats des Figs. 3 et 4, il apparaît maintenant que le degré d'hétérogénéité structurale interne du matériau (susceptible d'être défini avec précision) peut intervenir de façon dominante dans la création de l'état morphologique de surface. 


\section{Conclusions}

Le poids économique des phénomènes de surface tels que lubrification, frottement, usure, adhésion, mouillage, contacts élastiques et thermiques interfaciaux, intervenant dans les applications industrielles et les usages quotidiens, justifie l'intérêt croissant accordé à la caractérisation de l'état morphologique des surfaces. En effet, la connaissance de cet état permet de comprendre et parfois de prévoir le comportement statique ou dynamique des pièces en contact. Cet état morphologique dépend de deux ensembles de facteurs:

- les facteurs se rattachant aux procédés d'élaboration de la surface et qui se reconnaissent notamment par la "signature" mécanique des machines-outils,

- les facteurs de la physique du solide, auquels on s'est surtout intéressé ici, qui caractérisent les processus et l'état de structuration interne des matériaux.

Bien qu'ils puissent aider à la compréhension des formes observées, ces facteurs apparaissent encore trop négligés dans les réflexions prédictives de la morphologie des surfaces. En effet, on sait que les processus élémentaires d'interaction et de structuration à l'échelle atomique ou moléculaire (germination, croissance, ségrégation, transitions de phases, ...) constituent des singularités de comportement des systèmes physiques, à partir desquels se crée l'organisation macroscopique et continue de la matière et la morphogenèse des surfaces qui en découle.

La caractérisation morphologique des surfaces procède d'une analyse statistique des profils topographiques d'une région superficielle dans diverses directions. Elle s'appuie ainsi sur les outils de traitement et les grandeurs mis au point dans cette branche des mathématiques. Les paramètres de forme caractérisant l'aplatissement et l'asymétrie des profils s'avèrent particulièrement intéressants pour identifier les formes morphologiques. Ils ont permis de montrer que le degré d'hétérogénéité structurale interne des solides peut intervenir de façon dominante dans la création de l'état morphologique de surface.

Cependant, le lien entre la physique du solide et la morphologie des surfaces reste délicat à établir. En effet, il résulte d'une combinaison et d'un enchevêtrement entre les propriétés physico-chimiques et les propriétés mécaniques qui interagissent entre elles aux différents stades d'élaboration de la surface. L'exigence industrielle de surfaces de mieux en mieux définies, à des échelles de plus en plus petites (nanométriques et même subnanométriques), devrait permettre de mieux établir ces liens et d'améliorer nos capacités prédictives, car les phénomènes physiques de structuration de la matière interviendront alors plus directement dans les observations.

\section{Références}

[1] D. Marr, Vision, Freeman, San Francisco 1982.

[2] R. Thom, Stabilité Structurelle et Morphogénèse, Benjamin, New York, Ediscience, Paris 1972.

[3] R. Thom, SIAM Rev. 19, 189 (1977).

[4] H. Zahouani, T.G. Mathia, A. Guinet, Finnish J. Tribology 2, 125 (1992). 
[5] T.G. Mathia, J.L. Brugirard, J. Duarte, B. Maurin-Perrier, Surf. Topography 2, 157 (1989); G. Mathia, J.L. Brugirard, M. Balleydier, J. Duarte, B. Maurin-Perrier, Surf. Topography 2, 173 (1989); P. Kapsa, T. Mathia, A. Szuder, Rev. Roum. Sci. Techn. - Mec. Appl. 33, 277 (1988).

[6] H. Zahouani, T.G. Mathia, A. Guinet, Finnish J. Tribology 2, 125 (1992).

[7] C. Gauthier, Thèse de doctorat, Université de Nantes, Nantes 1988.

[8] A. Guinert, Thèse de doctorat, Ecole Centrale de Lyon, Lyon 1991.

[9] J.D.B. de Mello, M. Durand-Chare, T. Mathia, Maler. Sci. Eng. 73, 203 (1985); J.D.B. de Mello, M. Durand-Chare, Mater. Sci. Eng. 78, 127 (1986); J.D.B. de Mello, E. Lopez, T. Mathia, in: Annales du VI Congresso Latinoamericano Sobre Methodos Compulacionales - MECOM 85 Parana - Santa Fe (Argentine) 1985, Asociacion Argentina de Mecanica Computacional, 1985, p. 307. 